\title{
Strong oriented chromatic number of planar graphs without short cycles
}

\author{
Mickaël Montassier ${ }^{1}$, Pascal Ochem ${ }^{2}$, and Alexandre Pinlou ${ }^{3}$ \\ ${ }^{1}$ LaBRI, Université Bordeaux 1, 351 Cours de la Libération, 33405 Talence Cedex, France \\ mickael.montassier@labri.fr \\ ${ }^{2}$ LRI, Université Paris-Sud, 91405 Orsay Cedex, France \\ pascal.ochemelri.fr \\ ${ }^{3}$ LIRMM, Université Montpellier 2, 161 rue Ada, 34392 Montpellier Cedex 5, France \\ alexandre.pinloulirmm. fr
}

received Feb 08, 2006, revised Sep 18, 2007, accepted Dec 22, 2007.

Let $M$ be an additive abelian group. An $M$-strong-oriented coloring of an oriented graph $G$ is a mapping $\varphi$ from $V(G)$ to $M$ such that $\varphi(u) \neq \varphi(v)$ whenever $\overrightarrow{u v}$ is an arc in $G$ and $\varphi(v)-\varphi(u) \neq-(\varphi(t)-\varphi(z))$ whenever $\overrightarrow{u v}$ and $\overrightarrow{z t}$ are two arcs in $G$. The strong oriented chromatic number of an oriented graph is the minimal order of a group $M$ such that $G$ has an $M$-strong-oriented coloring. This notion was introduced by Nešetřil and Raspaud [Ann. Inst. Fourier, 49(3):1037-1056, 1999].

We prove that the strong oriented chromatic number of oriented planar graphs without cycles of lengths 4 to 12 (resp. 4 or 6 ) is at most 7 (resp. 19). Moreover, for all $i \geq 4$, we construct outerplanar graphs without cycles of lengths 4 to $i$ whose oriented chromatic number is 7 .

Keywords: Oriented graph, strong oriented coloring, discharging procedure, maximum average degree.

\section{Introduction}

Oriented graphs are directed graphs without loops nor opposite arcs. For two adjacent vertices $u$ and $v$, we denote by $\overrightarrow{u v}$ the arc from $u$ to $v$ or simply $u v$ whenever its orientation is not relevant (therefore, $u v=\overrightarrow{u v}$ or $u v=\overrightarrow{v u}$ ).

An oriented $k$-coloring of an oriented graph $G$ is a mapping $\varphi$ from $V(G)$ to a set of $k$ colors such that (1) $\varphi(u) \neq \varphi(v)$ whenever $\overrightarrow{u v}$ is an arc in $G$, and (2) $\varphi(u) \neq \varphi(x)$ whenever $\overrightarrow{u v}$ and $\overrightarrow{w x}$ are two arcs in $G$ with $\varphi(v)=\varphi(w)$. The oriented chromatic number of an oriented graph, denoted by $\chi_{o}(G)$, is defined as the smallest $k$ such that $G$ admits an oriented $k$-coloring.

Let $G$ and $H$ be two oriented graphs. A homomorphism from $G$ to $H$ is a mapping $\varphi: V(G) \rightarrow V(H)$ that preserves the arcs: $\overrightarrow{\varphi(x) \varphi(y)} \in A(H)$ whenever $\overrightarrow{x y} \in A(G)$.

An oriented $k$-coloring of $G$ can be equivalently defined as a homomorphism from $G$ to $H$, where $H$ is an oriented graph of order $k$. Then, the oriented chromatic number $\chi_{o}(G)$ of $G$ can be defined as the smallest order of an oriented graph $H$ such that $G$ admits a homomorphism to $H$. 
The notion of oriented coloring can be extended to undirected graphs saying that the oriented chromatic number $\chi_{o}(G)$ of an undirected graph $G$ is the maximum oriented chromatic number taken over all orientations of $G$.

The problem of bounding the oriented chromatic number has already been investigated for various graph classes: graphs with bounded maximum average degree [4], graphs with bounded degree [7], graphs with bounded treewidth [13, 14], and graph subdivisions [15].

Nešetřil and Raspaud [10] introduced the strong oriented chromatic number. Let $M$ be an additive abelian group. A strong oriented coloring of an oriented graph $G$ is a mapping $\varphi$ from $V(G)$ to $M$ such that $(1) \varphi(u) \neq \varphi(v)$ whenever $\overrightarrow{u v}$ is an arc in $G$ and $(2) \varphi(v)-\varphi(u) \neq-(\varphi(t)-\varphi(z))$ whenever $\overrightarrow{u v}$ and $\overrightarrow{z t}$ are two arcs in $G$ (this last condition will be called the no-opposite value condition). We say that $G$ has a $M$-strong-oriented coloring. The strong oriented chromatic number of an oriented graph, denoted by $\chi_{s}(G)$, is the minimal order of a group $M$, such that $G$ has $M$-strong-oriented coloring.

A strong oriented coloring of an oriented graph $G$ can be equivalently defined as a homomorphism $\varphi$ from $G$ to $H$, where $H$ is an oriented graph with $k$ vertices labeled by the $k$ elements of an abelian additive group $M$, such that for any pair of arcs $\overrightarrow{u v}$ and $\overrightarrow{z t}$ of $A(G), \varphi(v)-\varphi(u) \neq-(\varphi(t)-\varphi(z))$. Then, the strong oriented chromatic number of $G$ can be defined as the smallest order of an additive abelian group $M$ such that $G$ admits a homomorphism to $H$ labeled by the elements of $M$ satisfying the no-opposite value condition. Analogously, the strong oriented chromatic number of an undirected graph $G$ is the maximum strong oriented chromatic number taken over all the orientations of $G$.

Therefore, any strong oriented coloring of $G$ is an oriented coloring of $G$; hence, $\chi_{o}(G) \leq \chi_{s}(G)$.

Let $M$ be an additive group and let $S \subset M$ be a set of group elements. The Cayley digraph associated with $(M, S)$, denoted by $C_{(M, S)}$, is then defined as follows:

$$
V\left(C_{(M, S)}\right)=M \text { and } A\left(C_{(M, S)}\right)=\{(g, g+s) ; g \in M, s \in S\} .
$$

If the set $S$ are group generators of $M$, then $C_{(M, S)}$ is connected.

Assuming that $M$ is abelian and $S \cap-S=\emptyset$, then $C_{(M, S)}$ is oriented (neither loops nor opposite arcs), and for any pair $\left(g_{1}, g_{1}+s_{1}\right)$ and $\left(g_{2}, g_{2}+s_{2}\right)$ of arcs of $C_{(M, S)}, g_{1}+s_{1}-g_{1} \neq-\left(g_{2}+s_{2}-g_{2}\right)$. Thus, finding a strong oriented $k$-coloring of an oriented graph $G$ may be viewed as finding a homomorphism from $G$ to an oriented Cayley graph $C_{(M, S)}$ of order $k$, for some abelian group $M$ with $S \subset M$ and $S \cap-S=\emptyset$.

Let $H$ be an oriented graph. In the following, we say that an oriented graph $G$ admits an $H$-coloring whenever there exists a homomorphism from $G$ to $H$. In the undirected case, we say that an undirected graph $G$ admits an $H$-coloring when every orientation of $G$ admits a homomorphism to $H$.

Strong oriented coloring of planar graphs was recently studied. Sámal [12] proved that every oriented planar graph admits a strong oriented coloring with at most 672 colors. Marshall [8] improved this result and proved the following:

Theorem 1.1 [8] Let $G$ be an oriented planar graph. Then $\chi_{s}(G) \leq 271$. 
Borodin et al. [3, 4] studied the relationship between the strong oriented chromatic number and the maximum average degree of a graph, where the maximum average degree, denoted by $\operatorname{mad}(G)$ is:

$$
\operatorname{mad}(G)=\max \left\{\frac{2|A(H)|}{|V(H)|} ; H \subseteq G\right\}
$$

They considered homomorphisms to oriented Cayley graphs and proved that:

Theorem 1.2 [3, 4] Let $G$ be a graph.

1. If $\operatorname{mad}(G)<\frac{12}{5}$ and $G$ has girth at least 5 , then $\chi_{s}(G) \leq 5[3]$.

2. If $\operatorname{mad}(G)<\frac{11}{4}$ and $G$ has girth at least 5 , then $\chi_{s}(G) \leq 7$ [4].

3. If $\operatorname{mad}(G)<3$, then $\chi_{s}(G) \leq 11[4]$.

4. If $\operatorname{mad}(G)<\frac{10}{3}$, then $\chi_{s}(G) \leq 19$ [4].

The girth of a graph $G$ is the length of a shortest cycle of $G$. When considering planar graphs, the maximum average degree and the girth are linked by the following well-known relation:

Claim 1.3 [4] Let $G$ be a planar graph with girth $g$. Then, $\operatorname{mad}(G)<2+\frac{4}{g-2}$.

Corollary 1.4 follows from the previous claim.

Corollary 1.4 [3. 4] Let $G$ be a planar graph.

1. If $G$ has girth at least 12 , then $\chi_{s}(G) \leq 5[3]$.

2. If $G$ has girth at least 8 , then $\chi_{s}(G) \leq 7$ [4].

3. If $G$ has girth at least 6 , then $\chi_{s}(G) \leq 11$ [4]].

4. If $G$ has girth at least 5, then $\chi_{s}(G) \leq 19$ [4].

The following two theorems give upper bounds for planar graphs with girth 7 and 4, respectively. The proofs of these results do not use arguments on the maximum average degree.

Hence, Borodin and Ivanova [1] recently improved the previous result:

Theorem 1.5 [1] Let $G$ be a planar graph with girth at least 7. Then $\chi_{s}(G) \leq 7$.

Moreover, the class of triangle-free planar graphs was studied by Ochem [11], and by Borodin and Ivanova [2]:

Theorem 1.6 [2] Let $G$ be a triangle-free planar graph. Then $\chi_{s}(G) \leq 47$.

Notice that, for general graphs, the oriented chromatic number is not bounded when the maximum average degree tends to 4 [4]. Therefore, Theorem 1.6 cannot be obtained via the maximum average degree since triangle-free planar graphs have maximum average degree arbitrarily close to 4 . 
The best known upper bounds on the strong oriented chromatic number of planar graphs and trianglefree planar graphs are respectively 271 and 47. It seems that triangles play an important role for this invariant. In this paper, we study the strong oriented chromatic number of planar graphs without cycles of lengths 4 to $i$ for a some $i \geq 4$. These graph classes appear in Steinberg's conjecture [6]. The aim of this study is to check whether triangles have a significant influence on the strong oriented chromatic number of planar graphs.

Notice that the best known upper bounds on the strong oriented chromatic number of planar graphs with girth 5, 6, and 12 are obtained via the maximum average degree. Therefore, to get bounds on the strong oriented chromatic number of planar graphs without cycles of lengths 4 to $i, i \geq 4$, it is natural to determine the maximum average degree of these classes. The following two lemmas give tight bounds on the maximum average degree of planar graphs without cycles of lengths 4 to $i$ for all $i \geq 4$.

\section{Lemma 1.7}

1. If $G$ is a planar graph without cycles of length 4 , then $\operatorname{mad}(G)<\frac{30}{7}$.

2. For all $\epsilon>0$, there exists a planar graph $G$ without cycles of length 4 such that $\operatorname{mad}(G)>\frac{30}{7}-\epsilon$.

Lemma 1.8 For all $i \geq 5$,

1. If $G$ is a planar graph without cycles of lengths 4 to $i$, then $\operatorname{mad}(G)<3+\frac{3}{i-2}$.

2. For all $\epsilon>0$, there exists a planar graph $G$ without cycles of lengths 4 to $i$ such that $\operatorname{mad}(G)>$ $3+\frac{3}{i-2}-\epsilon$.

By Lemma 1.8 , every planar graph $G$ without cycles of lengths 4 to 11 has $\operatorname{mad}(G)<3+\frac{3}{11-2}=\frac{10}{3}$. Consequently, we obtain the following corollary by Theorem 1.24

Corollary 1.9 If $G$ is a planar graph without cycles of lengths 4 to 11 , then $\chi_{s}(G) \leq 19$.

Lemmas 1.72 and 1.82 show that, for every $i \geq 4$, there exist planar graphs $G$ without cycles of lengths 4 to $i$ such that $\operatorname{mad}(G)>3$. Thus, Theorem 1.2 cannot provide an upper bound less than 19 . In the remainder, we improve Corollary 1.9 and prove the following theorems:

Theorem 1.10 Let $G$ be planar graph without cycles of lengths 4 to 12. Then, $\chi_{s}(G) \leq 7$.

Theorem 1.11 For all $i \geq 4$, there exists an outerplanar graph $G$ without cycles of lengths 4 to $i$ such that $\chi_{o}(G) \geq 7$.

Theorem 1.12 Let $G$ be planar graph without cycles of lengths 4 or 6 . Then, $\chi_{s}(G) \leq 19$.

In the next section, we prove Lemmas 1.7 and 1.8 . In Section 3 , we introduce the Cayley tournaments $Q R_{7}$ and $Q R_{19}$ and some of their properties. Section 4 is dedicated to the proof of Theorem 1.10 , we prove that every oriented planar graph without cycles of lengths 4 to 12 has a homomorphism to the Cayley graph $Q R_{7}$. In Section 5, we prove Theorem 1.11 which shows that Theorem 1.10 is tight in some way. Section 6 is dedicated to the proof of Theorem 1.12, we show that every oriented planar graph without cycles of lengths 4 or 6 has a homomorphism to the Cayley graph $Q R_{19}$. In Section 7 , we give some concluding remarks and state recent related results. 
In the following, $V(G), A(G)$, and $F(G)$ denote respectively the sets of vertices, edges/arcs, and faces of a plane graph $G$. For a vertex $v$, we denote by $d^{-}(v)$ the indegree of $v$, by $d^{+}(v)$ its outdegree, and by $d(v)$ its degree. A vertex of degree $k$ (resp. at least $k$, at most $k$ ) is called a $k$-vertex (resp. $\geq k$-vertex, $\leq k$-vertex). The size of a face $f$, denoted by $d(f)$, is the number of edges on its boundary walk, where each cut-edge is counted twice. A face of $\operatorname{size} l$ (resp. at least $l$, at most $l$ ) is called a $l$-face (resp. ${ }^{l} l$-face, $\leq l$-face).

\section{Proofs of Lemmas 1.7 and 1.8}

In this section, we give the proofs of Lemmas 1.7 and 1.8 which characterize the maximum average degree of planar graphs without cycles of lengths 4 to $i$ for all $i \geq 4$.

\section{Proof of Lemma 1.7;}

1. Observe that we can rewrite Euler's formula $|V(G)|-|A(G)|+|F(G)|=2$ as follows:

$$
\sum_{v \in V(G)}(7 d(v)-30)+\sum_{f \in F(G)}(8 d(f)-30)=-60
$$

We define the weight function $\omega$ by $\omega(f)=8 d(f)-30$ for each face $f$ in $F(G)$. Now, we redistribute the weights according to the following discharging rule: every $\geq 5$-face gives 2 to each adjacent 3-face. Let $f$ be a face of $F(G)$ and let $\omega^{*}(f)$ denote its weight after discharging. If $d(f)=3$, then $\omega^{*}(f)=\omega(f)+3 \times 2=0$. If $d(f) \geq 5$, then $\omega^{*}(f) \geq \omega(f)-2 d(f)=$ $6 d(f)-30 \geq 0$. Since the total sum of weights is fixed by the discharging rule, we have:

$$
\sum_{f \in F(G)}(8 d(f)-30)=\sum_{f \in F(G)} \omega(f)=\sum_{f \in F(H)} \omega^{*}(f) \geq 0
$$

By Equation 1 , we have $\sum_{v \in V(G)}(7 d(v)-30)<0$, which implies that $\operatorname{mad}(G)<\frac{30}{7}$, since each subgraph of $G$ is a planar graph without cycles of length 4 .

2. We now prove that the upper bound proved above is tight. For all $\epsilon>0$, we construct a planar graph $G_{k}$ without cycles of length 4 such that $\operatorname{mad}\left(G_{k}\right)>\frac{30}{7}-\epsilon$.

Let $H$ be the graph of Fig. 1(a) This graph is planar and has no cycles of length 4 . Let $G_{k}$ be the graph obtained by tiling the plane with $k \times k$ copies of $H$ ( $k$ lines and $k$ columns); see Fig. 1(b),

We easily check that, for all $k \geq 1, G_{k}$ is planar and has no cycles of length 4 . The graph $G_{k}$ contains $k^{2}$ copies of $H$ and each copy contains 31 vertices and 54 edges. We can then compute that $\left|V\left(G_{k}\right)\right|=21 k^{2}+12 k-2$ and $\left|A\left(G_{k}\right)\right|=45 k^{2}+12 k-3$.Thus,

$$
\operatorname{mad}\left(G_{k}\right) \geq \frac{2\left|A\left(G_{k}\right)\right|}{\left|V\left(G_{k}\right)\right|}=\frac{2\left(45 k^{2}+12 k-3\right)}{21 k^{2}+12 k-2}=\frac{30}{7}-\frac{192 k+29}{147 k^{2}+84 k-14}
$$

This allows us to conclude:

$$
\forall \epsilon>0, \exists k \geq 1: \operatorname{mad}\left(G_{k}\right)>\frac{30}{7}-\epsilon .
$$




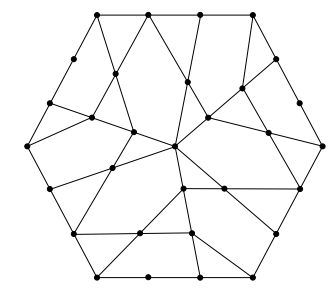

(a) The graph $H$.

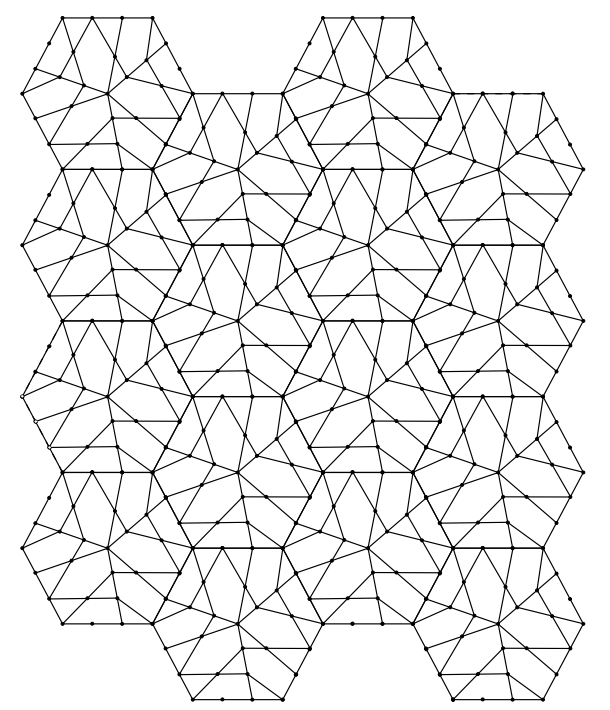

(b) The graph $G_{k}$.

Fig. 1: Tiling the plane to obtain a planar graph $G_{k}$ without cycles of length 4 such that $\operatorname{mad}\left(G_{k}\right)>\frac{30}{7}-\epsilon$.

\section{Proof of Lemma 1.8;}

1. Let $G$ be a counterexample to Lemma 1.811 which is minimal according to the subgraph order. In particular, $G$ is a planar graph without cycles of lengths 4 to $i$ with maximum average degree at least $3+\frac{3}{i-2}$. Moreover, by minimality, $G$ contains neither 1-vertices nor two adjacent 2-vertices. Let $G^{\prime}$ be the graph obtained from $G$ by removing every 2-vertex incident to a 3-face. Let $n, n_{2}$, $n_{3}$, and $n_{4}$ denote respectively the number of vertices, 2-vertices, 3-vertices, and $\geq 4$-vertices of $G^{\prime}$. An edge in $G^{\prime}$ is said to be free if it is not incident to a 3-face in $G^{\prime}$. Let $m, m_{3}$, and $m_{i}$ denote respectively the number of edges, edges incident to a 3-face, and free edges of $G^{\prime}$. Let $f, f_{3}$, and $f_{i}$ denote respectively the number of faces, 3 -faces, and $\geq(i+1)$-faces of $G^{\prime}$.

In $G^{\prime}$, a 2-vertex is incident to two free edges. Moreover, a 3-vertex is incident to at least one free edge, since otherwise $G^{\prime}$ would contain a cycle of length 4 . We thus have

$$
2 n_{2}+n_{3} \leq 2 m_{i}
$$

By definition:

$$
\begin{gathered}
n=n_{2}+n_{3}+n_{4} \\
m_{3}+m_{i}=m
\end{gathered}
$$




$$
f=f_{3}+f_{i}
$$

Since $G^{\prime}$ contains no cycle of length 4, we have:

$$
3 f_{3}=m_{3}
$$

Considering the sum of vertex degrees in $G^{\prime}$, we have:

$$
2 n_{2}+3 n_{3}+4 n_{4} \leq 2 m
$$

Considering the sum of face degrees in $G^{\prime}$, we have:

$$
3 f_{3}+(i+1) f_{i} \leq 2 m
$$

Considering the Euler's formula for $G^{\prime}$, we have:

$$
m+2=n+f
$$

Let $s$ denotes the number of 2-vertices incident to a triangle in $G$. This means that $G$ contains $n+s$ vertices and $m+2 s$ edges. By minimality of $G$, the maximum average degree of $G$ equals its average degree and is at least $3+\frac{3}{i-2}$. We thus have:

$$
\left(3+\frac{3}{i-2}\right)(n+s) \leq 2(m+2 s)
$$

If $w$ is a 2-vertex incident to a triangle $u v w$ in $G$, then $u v$ is a free edge in $G^{\prime}$. Moreover, if $u v$ is a free edge in $G^{\prime}$, there exists at most one 2-vertex $w$ such that uvw is a triangle in $G$ ( $G$ does not contain cycles of length 4 ). We thus have:

$$
s \leq m_{i}
$$

The combination $3 \times \mathbf{( 2 )}+12 \times(\mathbf{3})+2(i-2) \times \mathbf{( 4 )}+6(i+1) \times(\mathbf{5})+2(i-2) \times(\mathbf{6})+3 \times(\mathbf{7})+$ $6 \times(\mathbf{8})+6(i+1) \times(\mathbf{9})+2(i-2) \times \mathbf{( 1 0})+2(i-5) \times \mathbf{1 1})$ gives $12(i+1) \leq 0$. This contradiction proves Lemma 1.81

2. We now prove that the upper bound proved above is tight. For all $\epsilon>0$ and for all $i \geq 5$, we construct a planar graph $G_{k}^{\prime}$ without cycles of lengths 4 to $i$ such that $\operatorname{mad}\left(G_{k}^{\prime}\right)>3+\frac{3}{i-2}-\epsilon$.

Let $H^{\prime}$ be the graph obtained from two chains of intersecting triangles, one of length $\left\lfloor\frac{i-5}{2}\right\rfloor$ and the other of length $\left\lceil\frac{i-5}{2}\right\rceil$, arranged as depicted in Fig. 2(a). For all $i \geq 5$, this graph is planar and has no cycles of lengths 4 to $i$. Let $G_{k}^{\prime}$ be the graph obtained by tiling the plane with $k \times k$ copies of $H^{\prime}$ ( $k$ lines and $k$ columns); see Fig. 2(b)

We easily check that, for all $i \geq 5$ and for all $k \geq 1$, the graph $G_{k}^{\prime}$ is planar and has no cycles of lengths 4 to $i$. The graph $G_{k}^{\prime}$ contains $k^{2}$ copies of $H^{\prime}$ and each copy contains $2(i+1)$ vertices and 


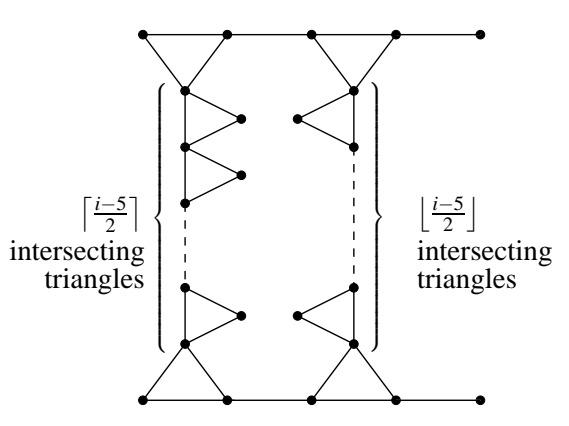

(a) The graph $H^{\prime}$.

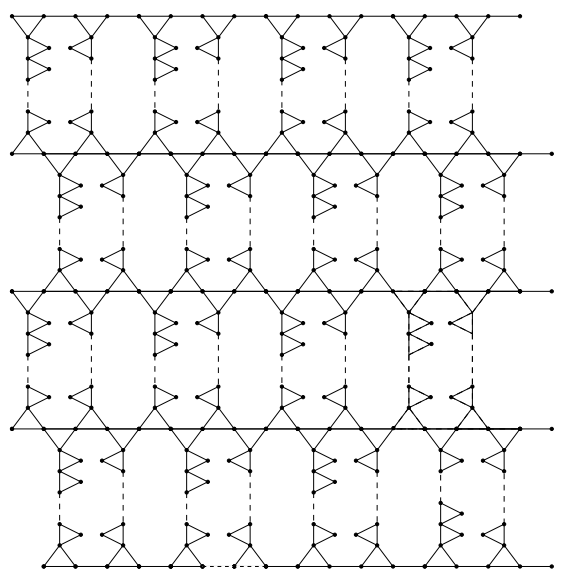

(b) The graph $G_{k}^{\prime}$.

Fig. 2: Tiling the plane to obtain a planar graph $G_{k}^{\prime}$ without cycles of lengths 4 to $i, i \geq 5$, such that $\operatorname{mad}\left(G_{k}^{\prime}\right)>$ $3+\frac{3}{i-2}-\epsilon$.

$3 i+1$ edges. We can then compute that $\left|V\left(G_{k}^{\prime}\right)\right|=2 i k^{2}-4 k^{2}+6 k$ and $\left|A\left(G_{k}^{\prime}\right)\right|=3 i k^{2}-3 k^{2}+$ $k-2$. Thus,

$$
\operatorname{mad}\left(G_{k}^{\prime}\right) \geq \frac{2\left|A\left(G_{k}^{\prime}\right)\right|}{\left|V\left(G_{k}^{\prime}\right)\right|}=\frac{6 i k^{2}-6 k^{2}+2 k-4}{2 i k^{2}-4 k^{2}+6 k}=3+\frac{3}{i-2}-\frac{8 i k-7 k+2 i-4}{k(i-2)(i k-2 k+3)}
$$

This allows us to conclude:

$$
\forall i \geq 5, \forall \epsilon>0, \exists k \geq 1: \operatorname{mad}\left(G_{k}^{\prime}\right)>3+\frac{3}{i-2}-\epsilon .
$$

\section{Some properties of the tournaments $Q R_{7}$ and $Q R_{19}$}

In this section, we define the tournaments $Q R_{7}$ and $Q R_{19}$, and give some properties that we use in next sections to prove Theorems 1.10 and 1.12

Recall that the circulant graph $G=C\left(p ; c_{1}, c_{2}, \ldots, c_{d}\right)$ is defined by $V(G)=\{0,1, \ldots, p-1\}$ and $\overrightarrow{u v} \in A(G)$ if and only if $v \equiv u+c_{i}(\bmod p)$ for some $i, 1 \leq i \leq d$. If $p \equiv 3(\bmod 4)$ is a prime power and the $c_{i}$ 's are the non zero quadratic residues modulo $p$, then $d=\frac{p-1}{2}$ and $Q R_{p}=C\left(p ; c_{1}, \ldots, c_{d}\right)$ is a Paley tournament.

Property 3.1 Let $p \equiv 3(\bmod 4), \mathbb{F}_{p}=\frac{\mathbb{Z}}{p \mathbb{Z}}$ and $S=\left\{x^{2} ; x \in \mathbb{F}_{p} \backslash\{0\}\right\}$ be the non zero squares of $\mathbb{F}_{p}$. Then $S \cap-S=\emptyset$. 


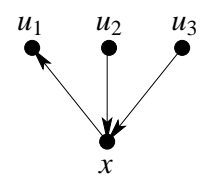

Fig. 3: $x$ is a $(0,1,1)$-successor of $\left(u_{1}, u_{2}, u_{3}\right)$

Proof: Since $p \equiv 3(\bmod 4),-1$ is not a square modulo $p$. Indeed, if it were, $-1=x^{2}$ and so by Fermat's little theorem: $(-1)^{\frac{(p-1)}{2}}=x^{p-1}=1$. But $(-1)^{\frac{(p-1)}{2}}=-1$.

Then, let $a, b \in \mathbb{F}_{p}$ such that $a^{2}+b^{2}=0$. If $a \neq 0$ or $b \neq 0$, then $\left(\frac{b}{a}\right)^{2}=-1$ or $\left(\frac{a}{b}\right)^{2}=-1$, which is a contradiction. Therefore, $a=b=0$.

Therefore, such a Paley tournament $Q R_{p}$ is clearly the Cayley graph $C_{(M, S)}$ with $M=\mathbb{F}_{p}=\frac{\mathbb{Z}}{p \mathbb{Z}}$ and $S=\left\{x^{2} ; x \in \mathbb{F}_{p} \backslash\{0\}\right\}$.

Another important property is that the $Q R_{p}$ tournaments are arc-transitive [5], which means that for every two arcs $\overrightarrow{u v}$ and $\overrightarrow{x y}$, there exists an automorphism mapping $\overrightarrow{u v}$ to $\overrightarrow{x y}$.

For instance, let us consider the tournaments $Q R_{7}=C(7 ; 1,2,4)$ and $Q R_{19}=C(19 ; 1,4,5,6,7,9$, $11,16,17)$, which are Paley tournaments on 7 and 19 vertices, respectively.

An orientation vector of length $n$ is an $n$-tuple $\alpha=\left(\alpha_{1}, \alpha_{2}, \ldots, \alpha_{n}\right)$ in $\{0,1\}^{n}$.

Let $G$ be an oriented graph and let $U=\left(u_{1}, u_{2}, \ldots, u_{n}\right)$ be a sequence of distinct vertices of $G$. For an orientation vector $\alpha$, a vertex $v$ of $G$ is said to be an $\alpha$-successor of $U$ if for every $i, 1 \leq i \leq n, \overrightarrow{v u_{i}}$ is an arc in $G$ if $\alpha_{i}=0$ and $\overrightarrow{u_{i} v}$ is an arc in $G$ otherwise (see Fig. 3 for an example).

We shall say that an oriented graph $G$ satisfies Property $P_{n, k}$ if for any sequence $U$ of $n$ pairwise distinct vertices of $G$ and any orientation vector $\alpha$ of length $n$, there exists at least $k$ vertices in $G$ which are $\alpha$-successors of $U$.

The tournaments $Q R_{7}$ and $Q R_{19}$ have the following properties:

Property 3.2 [4] The tournament $Q R_{7}$ satisfies Properties $P_{1,3}$ and $P_{2,1}$.

Property 3.3 [4] The tournament $Q R_{19}$ satisfies Properties $P_{1,9}, P_{2,4}$, and $P_{3,1}$.

Let us give an example; saying that $Q R_{7}$ has Property $P_{2,1}$ means that for any pair of distinct vertices $(x, y)$ of $Q R_{7}$ and any orientation vector $\alpha$ of length two (four possible orientation vectors), we have at least one $\alpha$-successor of $(x, y)$. For instance, for $x=0$ and $y=1$, we can check that there exists one $(0,0)$-successor (Fig. 4(a) , one (1,0)-successor (Fig. 4(b)], one (1,1)-successor (Fig. 4(c)) and two $(0,1)$-successors (Fig. 4(d) of $(x, y)$.

In the remainder of this section, we will consider some other properties of $Q R_{7}$.

For a given vertex $v$ of an oriented graph $G$, we denote by $\Gamma^{+}(v)$ (resp. $\Gamma^{-}(v)$ ) the set of outgoing neighbors of $v\left(\Gamma^{+}(v)=\{u \in V ; \overrightarrow{v u} \in A(G)\}\right)$ (resp. the set of incoming neighbors of $v\left(\Gamma^{-}(v)=\right.$ $\{u \in V ; \overrightarrow{u v} \in A(G)\})$ ). For a subset $S$ of vertices of $G$, we define:

$$
\Gamma^{+}(S)=\bigcup_{v \in S} \Gamma^{+}(v) \quad \text { and } \quad \Gamma^{-}(S)=\bigcup_{v \in S} \Gamma^{-}(v)
$$




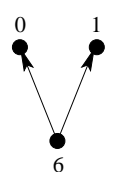

(a)

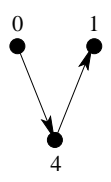

(b)

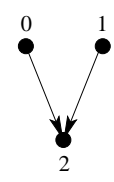

(c)

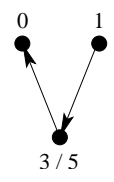

(d)

Fig. 4: Example of Property $P_{2,1}$ of $Q R_{7}$

For a given oriented graph $G$, we will denote by $\Omega_{G}(k)$ the largest number $n$ such that for all sets $S \subseteq V(G)$ of size $k,\left|\Gamma^{+}(S)\right| \geq n$ and $\left|\Gamma^{-}(S)\right| \geq n$.

By a case analysis, we have the following property:

\section{Property 3.4}

1. $\Omega_{Q R_{7}}(1)=3$;

2. $\Omega_{Q R_{7}}(2)=5$;

3. $\Omega_{Q R_{7}}(k)=6$ for $3 \leq k \leq 4$;

4. $\Omega_{Q R_{7}}(k)=7$ for $5 \leq k \leq 7$.

Let us consider the following definition.

Definition 3.5 Let $G$ be an oriented graph. Let $W \subseteq V(G)$ and $x_{1}, x_{2}, \ldots, x_{n}, y_{1}, y_{2}, \ldots, y_{m} \in W$ be $n+m$ vertices. Let $\gamma$ be a $Q R_{7}$-coloring of $G \backslash W . \gamma$ is called $\left(k_{1}, k_{2}, \ldots, k_{n} \mid l_{1}, l_{2}, \ldots, l_{m}\right)$ extendable to $\left(x_{1}, x_{2}, \ldots, x_{n} \mid y_{1}, y_{2}, \ldots, y_{m}\right)$ in $G$ if there exist $r=k_{1} \times k_{2} \times \ldots \times k_{n} Q R_{7}$-colorings $\gamma_{1}, \gamma_{2}, \ldots, \gamma_{r}$ of $G$ extending $\gamma$ such that

1. for all $1 \leq j \leq n,\left|\left\{\gamma_{i}\left(x_{j}\right) ; 1 \leq i \leq r\right\}\right|=k_{j}$,

2. the $r n$-tuples $\left(\gamma_{i}\left(x_{1}\right), \gamma_{i}\left(x_{2}\right), \ldots, \gamma_{i}\left(x_{n}\right)\right)$ are distinct,

3. for all $1 \leq j \leq m,\left|\left\{\gamma_{i}\left(y_{j}\right) ; 1 \leq i \leq r\right\}\right| \leq l_{j}$.

The $k_{j}$ colors for $x_{j}$ will be called choices since, for each $1 \leq j \leq n$, we can independently choose a color for each $x_{j}$ among the $k_{j}$ available colors, and then take the corresponding $Q R_{7}$-coloring $\gamma_{i}$ accordingly.

The $l_{j}$ colors for $y_{j}$ will be called possibilities since, for each $1 \leq j \leq m$, our only knowledge is that $y_{j}$ will have a color taken among at most $l_{j}$ colors.

Whenever we do not have informations about possibilities for some vertices, we will say that $\gamma$ is $\left(k_{1}, k_{2}, \ldots, k_{n}\right)$-extendable to $\left(x_{1}, x_{2}, \ldots, x_{n}\right)$ in $G$.

The drawing conventions for a configuration $C$ contained in a graph $G$ are the following. If $u$ and $v$ are two vertices of $C$, then they are adjacent in $G$ if and only if they are adjacent in $C$. Moreover, the neighbors of a white vertex in $G$ are exactly its neighbors in $C$, whereas a black vertex may have neighbors outside of $C$. Two or more black vertices in $C$ may coincide in a single vertex in $G$, provided they do not share a common white neighbor. Finally, an edge will represent an arc with any of its two possible orientations. 


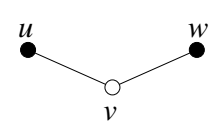

(a)

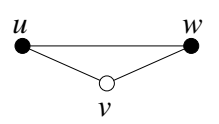

(b)

Fig. 5: Configurations of Properties 3.6 and 3.10

Property 3.6 Let $G$ be an oriented graph containing the configuration (a) of Fig. 5. let $W \subset V(G)$ and let $u, v, w \in W$ ( $u$ and $w$ are non-adjacent). Let $\gamma$ be a $Q R_{7}$-coloring of $G \backslash W$. If $\gamma$ is $(k, l)$-extendable to $(u, w)$ in $G \backslash\{v\}$, then it is $\left(\Omega_{Q R_{7}}(l)+\Omega_{Q R_{7}}(k)-7 \mid k, l\right)$-extendable to $(v \mid u, w)$ in $G$.

Proof: The $k$ choices of colors for $u$ allow $\Omega_{Q R_{7}}(k)$ distinct colors for $v$, while the $l$ choices of colors of $w$ allow $\Omega_{Q R_{7}}(l)$ distinct colors for $v$. By the Pigeon Hole Principle, we have at least $n=\Omega_{Q R_{7}}(k)+$ $\Omega_{Q R_{7}}(l)-7$ choices to color $v$ in $G$ while $u$ and $w$ have $k$ and $l$ possibilities, respectively.

A quick case study shows that the field $\mathbb{F}_{7}$ has the following property:

Property 3.7 Let $S=\left\{x^{2} ; x \in \mathbb{F}_{7} \backslash\{0\}\right\}=\{1,2,4\}$. For any two distinct couples $\left(s_{1}, s_{1}^{\prime}\right)$ and $\left(s_{2}, s_{2}^{\prime}\right)$ of $S$, we have $s_{1}-s_{1}^{\prime} \neq s_{2}-s_{2}^{\prime}$.

Property 3.7 allows us to prove the following:

Property 3.8 Let $x, y \in \mathbb{F}_{7}$ with $x \neq y$ and let $s_{1}, s_{2}, s_{3}, s_{4} \in S=\left\{x^{2} ; x \in \mathbb{F}_{7} \backslash\{0\}\right\}$ such that $s_{1} \neq s_{2}$ and $s_{3} \neq s_{4}$. Then $\left|\left\{x+s_{1}, x+s_{2}, y+s_{3}, y+s_{4}\right\}\right| \geq 3$ and $\left|\left\{x-s_{1}, x-s_{2}, y-s_{3}, y-s_{4}\right\}\right| \geq 3$.

Proof: Suppose that $\left|\left\{x+s_{1}, x+s_{2}, y+s_{3}, y+s_{4}\right\}\right|<3$. Since $s_{1} \neq s_{2}$ and $s_{3} \neq s_{4}$, we have $x+s_{1} \neq x+s_{2}$ and $y+s_{3} \neq y+s_{4}$. Therefore, we may assume w.l.o.g. that $x+s_{1}=y+s_{3}$ and $x+s_{2}=y+s_{4}$, which implies that $s_{3}-s_{1}=s_{4}-s_{2}$, that is a contradiction by Property 3.7

The same argument allows us to prove that $\left|\left\{x-s_{1}, x-s_{2}, y-s_{3}, y-s_{4}\right\}\right| \geq 3$.

A careful case study of $Q R_{7}$ gives the following:

Property 3.9 Let $x, y, z$ be three distinct vertices of $Q R_{7}$ such that $\overrightarrow{x y} \in A\left(Q R_{7}\right)$ and $\overrightarrow{x z} \in A\left(Q R_{7}\right)$. For any orientation vector $\alpha=\left(\alpha_{1}, \alpha_{2}\right) \in\{0,1\}^{2}$, there exist at least two distinct vertices $y^{\prime}$ and $z^{\prime}$ such that $y^{\prime}$ is an $\alpha$-successor of $(x, y)$ and $z^{\prime}$ is an $\alpha$-successor of $(x, z)$.

Property 3.10 Let $G$ be an oriented graph containing the configuration (b) of Fig. 5 let $W \subseteq V(G)$, and let $u, v, w \in W$ ( $u$ and $w$ are adjacent). Let $\gamma$ be a $Q R_{7}$-coloring of $G \backslash W$. If $\gamma$ is $(2 \mid 1)$-extendable (resp. (5 3$)$-extendable) to $(u \mid w)$ in $G \backslash\{v\}$, then $\gamma$ is $(2 \mid 2,1)$-extendable (resp. $(3 \mid 5,3)$-extendable) to $(v \mid u, w)$ in $G$.

Proof: Let $G^{\prime}=G \backslash\{v\}$ and we may assume w.l.o.g. that $\overrightarrow{w u} \in A(G)$.

1. Suppose that $\gamma$ is $(2 \mid 1)$-extendable to $(u \mid w)$ in $G^{\prime}$. In other words, there exist at least two $Q R_{7}$ colorings $\gamma_{1}$ and $\gamma_{2}$ of $G^{\prime}$ extending $\gamma$ such that $\gamma_{1}(u) \neq \gamma_{2}(u)$ and $\gamma_{1}(w)=\gamma_{2}(w)$. Therefore, $\gamma_{1}(u)$ and $\gamma_{2}(u)$ are two distinct successors of $\gamma_{1}(w)$ in $Q R_{7}$. By Property 3.9 , we have at least two choices to color $v$ in $G$ while $u$ and $w$ have 2 and 1 possibilities, respectively. 


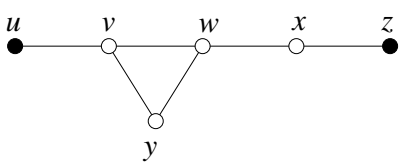

Fig. 6: Configuration of Property 3.11

2. Suppose now that $\gamma$ is $(5 \mid 3)$-extendable to $(u \mid w)$ in $G^{\prime}$. In other words, there exist at least five $Q R_{7}$-colorings $\gamma_{1}, \ldots, \gamma_{5}$ of $G^{\prime}$ extending $\gamma$ such that $\gamma_{i}(u)^{\prime} s$ are pairwise distinct and $\mid\left\{\gamma_{i}(w)\right.$; $1 \leq i \leq 5\} \mid=3$ (which is the worst case). Let $\gamma_{i}(u)=u_{i}$ and $\left\{\gamma_{i}(w) ; 1 \leq i \leq 5\right\}=$ $\left\{w_{1}, w_{2}, w_{3}\right\}$. The color $u_{i}$ is a successor of $\gamma_{i}(w)$ in $Q R_{7}$ for $1 \leq i \leq 5$. W.l.o.g, if there exist $j, k, l \in[1,5]$ with $j \neq k \neq l \neq j$ such that $\gamma_{j}(w)=\gamma_{k}(w)=\gamma_{l}(w)=w_{1}$, then $u_{j}, u_{k}, u_{l}$ are the three successors of $w_{1}$ in $Q R_{7}$ and by Property 3.9, we have at least three choices to color $v$ while $u$ and $w$ have 5 and 3 possibilities, respectively; thus $\gamma$ is $(3 \mid 5,3)$-extendable to $(v \mid u, w)$ in $G$. If it is not, by the Pigeon Hole Principle, we may assume w.l.o.g. that $u_{1}$ and $u_{2}$ are both successors of $w_{1}$ in $Q R_{7}$ and $u_{3}$ and $u_{4}$ are both successors of $w_{2}$ in $Q R_{7}$. By Property 3.9 for any orientation vector $\alpha \in\{0,1\}^{2}$, there exist $s_{1}, s_{2}, s_{3}, s_{4} \in S=\{1,2,4\}$ such that $w_{1}+s_{1}$ (resp. $\left.w_{1}+s_{2}, w_{2}+s_{3}, w_{2}+s_{4}\right)$ is an $\alpha$-successor of $\left(w_{1}, u_{1}\right)$ (resp. $\left(w_{1}, u_{2}\right),\left(w_{2}, u_{3}\right)$ and $\left.\left(w_{2}, u_{4}\right)\right)$ such that $w_{1}+s_{1} \neq w_{1}+s_{2}$ and $w_{2}+s_{3} \neq w_{2}+s_{4}$. Therefore, by Property 3.8 . $\left|\left\{w_{1}+s_{1}, w_{1}+s_{2}, w_{2}+s_{3}, w_{2}+s_{4}\right\}\right| \geq 3$ and we have at least three choices to color $v$ in $G$ while $u$ and $w$ have 5 and 3 possibilities, respectively; thus $\gamma$ is $(3 \mid 5,3)$-extendable to $(v \mid u, w)$ in $G$.

Property 3.11 Let $G$ be an oriented graph containing the configuration depicted on Fig. 6 and let $\gamma$ be a $Q R_{7}$-coloring of $G \backslash\{v, w, x, y\}$. Then, $\gamma$ is (3)-extendable to $(y)$ in $G$.

Proof: By Property 3.4 $\gamma$ is $(3,3)$-extendable to $(u, x)$ in $G \backslash\{w, y\}$. Then, by Property 3.6, $\gamma$ is $(5 \mid 3)$-extendable to $(w \mid u)$ in $G \backslash\{y\}$. Finally, by Property $3.10, \gamma$ is (3)-extendable to $(y)$ in $G$.

We will extensively use these previous properties in the next two sections to prove Theorems 1.10 and 1.12

\section{Proof of Theorem 1.10}

In this section, we prove that every oriented planar graph without cycles of lengths 4 to 12 has a homomorphism to the Cayley graph $Q R_{7}$.

Let $H$ be a counterexample to Theorem 1.10 of minimum order. We will show that in the following claim, $H$ does not contain some configurations. For each of them, we consider a reduction $H^{\prime}$ such that $\left|V\left(H^{\prime}\right)\right|<|V(H)|$. By minimality of $H$, there exists a $Q R_{7}$-coloring of $H^{\prime}$ and we show how to extend it to a $Q R_{7}$-coloring of $H$. Finally, a discharging procedure will complete the proof. 


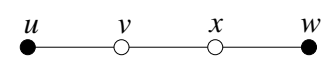

(a)

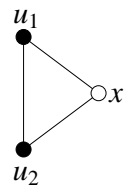

(b)

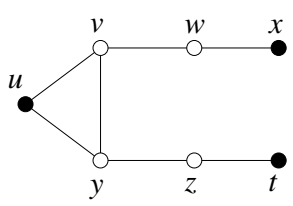

(c)

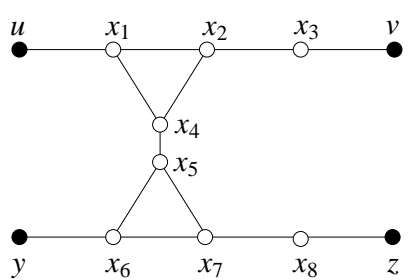

(d)

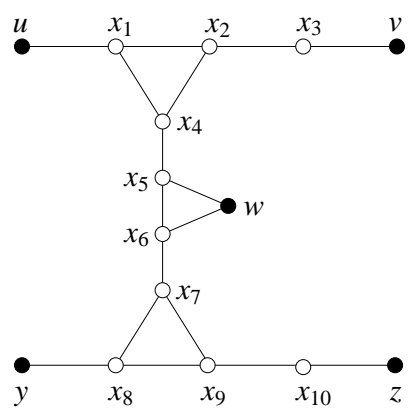

(e)

Fig. 7: Forbidden configurations of Theorem 1.10

\subsection{Structural properties of $H$}

Claim 4.1 The counterexample $H$ does not contain the following:

(C1) 1-vertices;

(C2) two adjacent 2-vertices;

(C3) a 3-face incident to a 2-vertex;

(C4) a 3-face incident to two 3-vertices, each of them adjacent to a 2-vertex;

(C5) the configuration depicted in Fig. 7 d);

(C6) the configuration depicted in Fig. 7 ( e).

Proof:

(C1) Trivial.

(C2) Consider Configuration (a) depicted in Fig. 7 Any $Q R_{7}$-coloring $\gamma$ of $H \backslash\{x\}$ can be modified such that $\gamma(v) \neq \gamma(w)$ thanks to Property $P_{1,3}$. This modified coloring can be extended to $H$ by Property $P_{2,1}$. 
(C3) Consider Configuration (b) depicted in Fig. 7. Any $Q R_{7}$-coloring $\gamma$ of $H \backslash\{x\}$ satisfies $\gamma\left(u_{1}\right) \neq$ $\gamma\left(u_{2}\right)$, and can be extended to $H$ by Property $P_{2,1}$.

(C4) Consider Configuration (c) depicted in Fig. 7 Let $\gamma$ be a $Q R_{7}$-coloring of $H \backslash\{v, w, y, z\}$. By Property $P_{1,3}$, we have two available colors to color the vertex $v$ such that $\gamma(v) \neq \gamma(x)$ in $H \backslash$ $\{w, y, z\}$. We choose the one such that by Property $P_{2,1}$, we can color the vertex $y$ with a color $\gamma(y) \neq \gamma(t)$ in $H \backslash\{w, z\}$. By Property $P_{2,1}$, we can finally extend $\gamma$ to a $Q R_{7}$-coloring of $H$.

(C5) Consider Configuration (d) depicted in Fig. 7. Let $\gamma$ be a $Q R_{7}$-coloring of $H \backslash\left\{x_{1}, \ldots, x_{8}\right\}$. By Property 3.11 $\gamma$ is $(3,3)$-extendable to $\left(x_{4}, x_{5}\right)$ in $H \backslash\left\{x_{4} x_{5}\right\}$. By Property 3.4 , the three choices of $x_{4}$ forbid at most one color for $x_{5}$ and therefore, there exist at least two couples of colors to color $x_{4}$ and $x_{5}$, i.e. there exist at least two $Q R_{7}$-coloring of $G$ extending $\gamma$.

(C6) Consider Configuration (e) depicted in Fig. 7. Let $\gamma$ be a $Q R_{7}$-coloring of $H \backslash\left\{x_{1}, \ldots, x_{10}\right\}$. By Property 3.11, $\gamma$ is $(3,3)$-extendable to $\left(x_{4}, x_{7}\right)$ in $H \backslash\left\{x_{5}, x_{6}\right\}$. Then, by Property 3.6 $\gamma$ is $(3,2)$-extendable to $\left(x_{4}, x_{6}\right)$ in $H \backslash\left\{x_{5}\right\}$. Then by Property $3.10 \gamma$ is $(3,2)$-extendable to $\left(x_{4}, x_{5}\right)$ in $H \backslash\left\{x_{4} x_{5}\right\}$. By Property 3.4, the three choices of $x_{4}$ forbid at most one color for $x_{5}$ and therefore, there exists at least one couple of colors to color $x_{4}$ and $x_{5}$, i.e. there exists at least one $Q R_{7}$-coloring of $G$ extending $\gamma$.

\subsection{Discharging procedure}

Lemma 4.2 Let $H$ be a connected plane graph. Then the following holds:

$$
\sum_{v \in V(H)}(11 d(v)-26)+\sum_{f \in F(H)}(2 d(f)-26)=-52
$$

Proof: Euler's formula $|V(G)|-|A(G)|+|F(G)|=2$ can be rewritten as $(22|A(G)|-26|V(G)|)+$ $(4|A(G)|-26|F(G)|)=-52$. This identity and the relation $\sum_{v \in V} d(v)=\sum_{f \in F} d(f)=2|A(G)|$ complete the proof.

A 3 -face $f$ incident to vertices $u, v$, and $w$ is light if each of $u, v$, and $w$ has degree 3 , and one of $u, v$, and $w$, say $w$, is adjacent to a 2-vertex. We say $w$ is weak, and $u$ and $v$ are light; see Fig. 8

We define the weight function $\omega$ by $\omega(x)=11 \cdot d(x)-26$ if $x \in V(H)$ and $\omega(x)=2 \cdot d(x)-26$ if $x \in F(H)$. It follows from Identity 12 that the total sum of weights is equal to -52 . In what follows, we will define discharging rules (R1) and (R2) and redistribute weights accordingly. Once the discharging is finished, a new weight function $\omega^{*}$ is produced. However, the total sum of weights is fixed by the discharging rules. Nevertheless, we can show that $\omega^{*}(x) \geq 0$ for all $x \in V(H) \cup F(H)$. This leads to the following obvious contradiction:

$$
0 \leq \sum_{x \in V(H) \cup F(H)} \omega^{*}(x) \leq \sum_{x \in V(H) \cup F(H)} \omega(x)=-52<0
$$

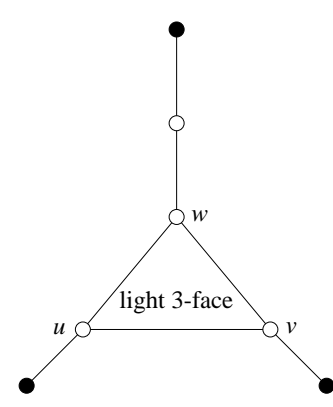

Fig. 8: A light 3-face 
That demonstrates that no such counterexample can exist.

The discharging rules are defined as follows:

(R1) Let $v$ be a $\geq 4$-vertex. (see Fig. 9 )

(R1.1) $v$ gives $\frac{1}{2}$ to each adjacent light 3-vertex.

(R1.2) $v$ gives 2 to each adjacent 2-vertex.

(R1.3) $v$ gives 9 to each incident 3-face.

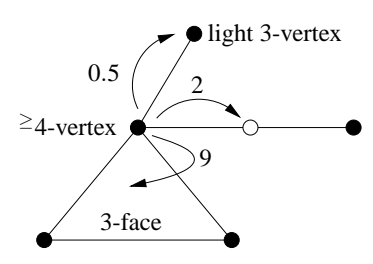

(R2) Let $v$ be a 3-vertex.

(R2.1) $v$ is incident to a light 3-face. (see Fig. 10)

Fig. 9: Rule (R1)

(R2.1.1) If $v$ is light, then $v$ gives $\frac{15}{2}$ to the incident light 3-face.

(R2.1.2) If $v$ is weak, then $v$ gives 2 to the adjacent 2-vertex and 5 to the incident light 3 -face.

(R2.2) $v$ is incident to 3-face which is not light. (see Fig. 11)

(R2.2.1) If $v$ is adjacent to a 2-vertex then $v$ gives 2 to the 2 -vertex and 5 to the incident 3 -face.

(R2.2.2) If $v$ is adjacent to a light 3-vertex, then $v$ gives $\frac{1}{2}$ to the light 3 -vertex and $\frac{13}{2}$ to the incident 3 -face.

(R2.2.3) In the other cases, $v$ gives 7 to the incident 3-face.

(R2.3) $v$ is not incident to a 3-face. Then $v$ gives $\frac{1}{2}$ to each adjacent light 3 -vertex and 2 to each adjacent 2 -vertex. (see Fig. 12)

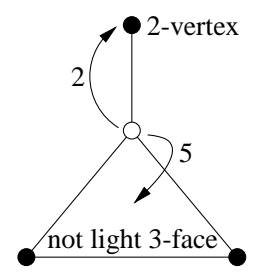

(a) Rule (R2.2.1)

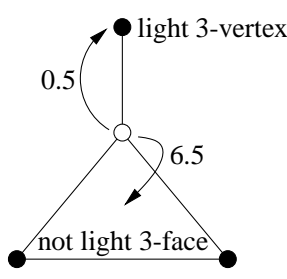

(b) Rule (R2.2.2)

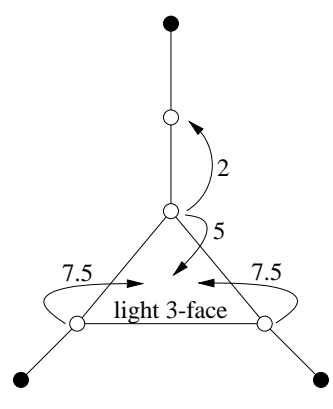

Fig. 10: Rule (R2.1)

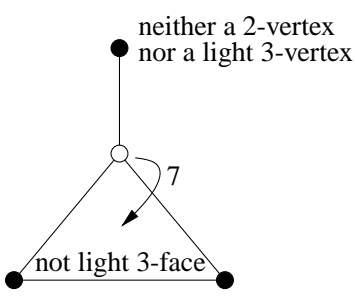

(c) Rule (R2.2.3)

Fig. 11: Rule (R2.2) 
Let $v$ be a $k$-vertex with $k \geq 2$ by (C1).

- If $k=2$, then $\omega(v)=-4$. Since two 2-vertices cannot be adjacent by $(\mathrm{C} 2), v$ receives 2 from each neighbor by $\mathrm{R} 1.2$, R2.1.2, R2.2.1 and R2.3. Hence, $\omega^{*}(v)=0$.

- If $k=3$, then $\omega^{*}(v)=7$. Suppose first that $v$ is not incident to a 3-face. By R2.3, $v$ gives at most $3 \cdot 2$ and $\omega^{*}(v) \geq 1$. Suppose now that $v$ is incident to a 3 -face $f$. If $f$ is light, then $v$ is light or weak. If $v$ is light then it receives $\frac{1}{2}$ from its neighbor which is not weak nor light by R1 and R2.3 (this

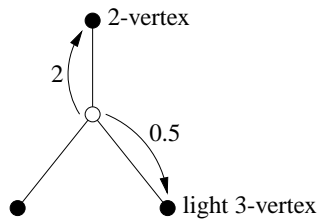

Fig. 12: Rule (R2.3) neighbor exists, since a light 3-vertex cannot be adjacent to two other light 3-vertices by (C5)). Then $v$ gives at most $\frac{15}{2}$ to $f$ by R2.1.1. Hence, $\omega^{*}(v)=7+\frac{1}{2}-\frac{15}{2}=0$. If $v$ is weak, by R2.1.2, it gives 7 and $\omega^{*}(v)=0$. If $f$ is not light, $v$ gives 7 by R2.2.1, R2.2.2 and R2.2.3, and $\omega^{*}(v)=0$.

- If $k \geq 4$, then $\omega(v)=11 \cdot k-26$. It is easy to observe that $v$ gives at most $\frac{9}{2} \cdot k$ by R1. Hence, $\omega^{*}(v) \geq 11 \cdot k-26-\frac{9}{2} \cdot k=\frac{13}{2} \cdot k-26 \geq 0$.

Let $f$ be an $l$-face. If $l \geq 13$, then $\omega(f)=\omega^{*}(f) \geq 0$. Suppose that $l=3 ; \omega(f)=-20$. If $f$ is light, then it receives $\frac{15}{2}$ from each incident light 3 -vertex and 5 from the incident weak vertex by R2.1.1 and R2.1.2, and $\omega^{*}(f)=-20+2 \cdot \frac{15}{2}+5=0$. Suppose that $f$ is not light. By (C3), $f$ is not incident to a 2 -vertex. If $f$ is incident to three 3 -vertices, then the vertices on the boundary of $f$ are adjacent to at most one light 3-vertex by (C6) and to no 2 -vertex by (C4); so $f$ receives at least $2 \cdot 7+\frac{13}{2}$ by R2.2.2 and R2.2.3. Hence, $\omega^{*}(v) \geq \frac{1}{2}$. Finally, if $f$ is incident to at least one $\geq 4$-vertex (therefore $f$ is not light), then $f$ receives at least $9+5+\frac{13}{2}$ by (C4), R1.3, R2.2.1, R2.2.2 and R2.2.3. Hence, $\omega^{*}(v) \geq \frac{1}{2}$.

For all $x \in V(H) \cup F(H), \omega^{*}(x) \geq 0$ which completes the proof of Theorem 1.10

\section{Proof of Theorem 1.11}

In this section, we prove that, for all $i \geq 4$, there exists an outerplanar graph $G$ without cycle of lengths 4 to $i$ such that $\chi_{o}(G) \geq 7$, which implies $\chi_{s}(G) \geq 7$. This result shows that the upper bound of Theorem 1.10 is tight for planar and outerplanar graphs. To get the required result, we need the two following lemmas.

Lemma 5.1 For all $n \geq 5, n \not \equiv 4(\bmod 5)$, we have $\chi_{o}\left(H_{n}\right) \geq 6$ (where $H_{n}$ is the graph depicted on Fig. 13).

Proof: The graph $H_{n}$ needs at least 5 colors for any oriented coloring: indeed, the vertices $u_{n}, v_{n}, u_{0}, v_{0}, u_{1}$ must have distinct colors. Suppose that $H_{n}$ admits a 5-oriented-coloring $f$ and let w.l.o.g. $f\left(u_{n}\right)=0, f\left(v_{n}\right)=1, f\left(u_{0}\right)=2, f\left(v_{0}\right)=3$ et $f\left(u_{1}\right)=4$. Then, we have two available colors, namely 0 and 1 , to color $v_{1}$ and $u_{2}$. However, $f\left(u_{n}\right)=0$ and $f\left(v_{n}\right)=1$; so we must set $f\left(v_{1}\right)=0$ and $f\left(u_{2}\right)=1$. Then, we have two available colors, namely 2 and 3 , to color $v_{2}$ and $u_{3}$.

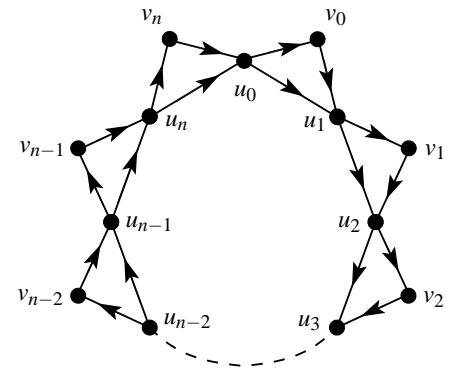

Fig. 13: An outerplanar graph $H_{n}$ without cycles of lengths 4 to $n$ such that $\chi_{o}\left(H_{n}\right) \geq 6$ for all $n \geq 5, n \not \equiv 4$ $(\bmod 5))$. 
However, $f\left(u_{0}\right)=2$ and $f\left(v_{0}\right)=3$; so, we must set $f\left(v_{2}\right)=2$ and $f\left(u_{3}\right)=3$. Finally, we have two available colors, namely 0 and 4 , to color $v_{3}$ and $u_{4}$. However, $f\left(u_{1}\right)=4$ and $f\left(v_{1}\right)=0$; so, we must set $f\left(v_{3}\right)=4$ and $f\left(u_{4}\right)=0$. It is then obvious that any 5-oriented-coloring of $H_{n}$ is a $T_{5}$-oriented-coloring (where $T_{5}$ is the tournament depicted on Fig. 14p. Now, we can check that for any $T_{5}$-oriented-coloring of $H_{n}$, we have $f\left(u_{i+1}\right) \equiv f\left(u_{i}\right)+2$ $(\bmod 5)$ (subscripts are taken modulo $n+1)$. Therefore, we have $f\left(u_{n}\right) \equiv f\left(u_{0}\right)+2 n(\bmod 5)$, and $f\left(u_{0}\right) \equiv f\left(u_{n}\right)+2(\bmod 5)$. Thus, $2+2 n \equiv 0(\bmod 5)$, what implies $n \equiv 4(\bmod 5)$, a contradiction. Therefore, $\chi_{o}\left(H_{n}\right) \geq 6$.

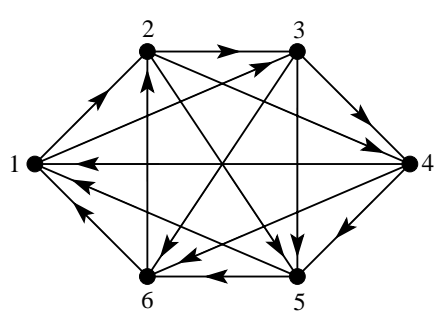

(a) $T_{6}^{1}$.

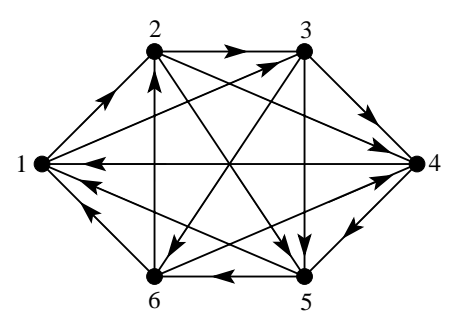

(b) $T_{6}^{2}$.

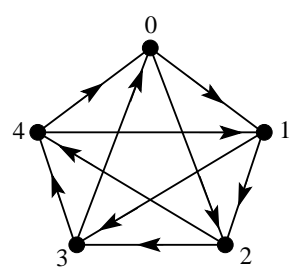

Fig. 14: The tournament $T_{5}$.

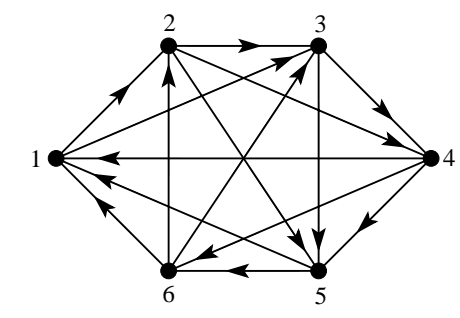

(d) $T_{6}^{4}$.

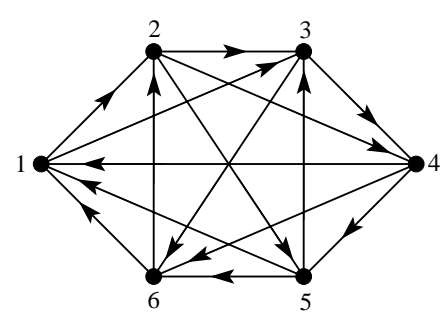

(c) $T_{6}^{3}$.

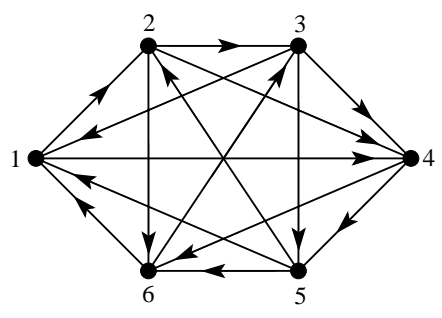

(e) $T_{6}^{5}$.

Fig. 15: The five non-isomorphic tournaments on six vertices such that for each vertex $u, d^{-}(u) \geq 2$ et $d^{+}(u) \geq 2$.

Let $T_{6}^{1}, T_{6}^{2}, T_{6}^{3}, T_{6}^{4}, T_{6}^{5}$ be the five tournaments on six vertices depicted on Fig. 15 .

Lemma 5.2 Let $n$ be an even integer and $H_{n}^{\prime}$ be the outerplanar graph depicted on Fig. 16 Any $T_{6}^{1}$ oriented-coloring (resp. $\left.T_{6}^{2}, T_{6}^{3}, T_{6}^{4}, T_{6}^{5}\right)$ f of $H_{n}^{\prime}$ is such that $f\left(w_{0}\right) \neq 1$.

Proof: Let $i \in[1,5]$. Suppose that the graph $H_{n}^{\prime}$ admits a $T_{6}^{i}$-oriented-coloring $f$ such that $f\left(w_{0}\right)=1$. Then, it is easy to check that, for all $i, 0 \leq i \leq n$, we have $f\left(w_{i}\right)=1$ if $i$ is even, and $f\left(w_{i}\right)=2$ otherwise. Therefore, we have $f\left(w_{n}\right)=f\left(w_{0}\right)$, that is forbidden. 


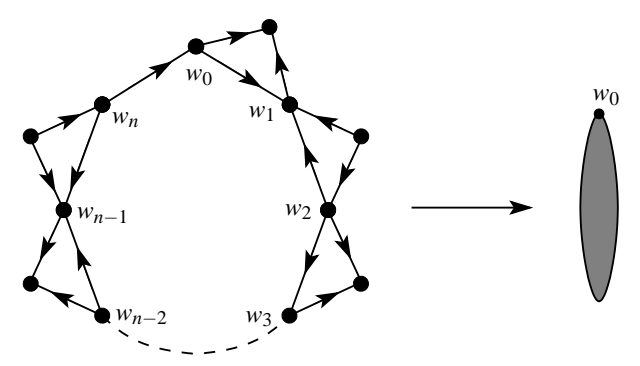

Fig. 16: An outerplanar graph $H_{n}^{\prime}$ without cycles of lengths 4 to $n$.

Proof of Theorem 1.11: Let $n \geq 5, n \not \equiv 4(\bmod 5)$, and $J_{n}$ be the outerplanar graph constructed as follows: we get $H_{n}$ (depicted in Fig. 13), $n+1$ copies of $H$ (depicted in Fig. 17) and we identify the vertex $x$ of each copy of $H$ to vertices $v_{0}, v_{1}, \ldots, v_{n}$. By Lemma 5.1 . $\chi_{o}\left(H_{n}\right) \geq 6$. Let $W=\left\{u_{1}, u_{2}, \ldots, u_{n}, v_{0}, v_{1}, \ldots, v_{n}\right\}$. Thus, if $\chi_{o}\left(J_{n}\right)=6$, the six colors are necessarily used on the vertices of $W$ of $J_{n}$. In addition, since each vertex of $W$ has two successors and two predecessors with necessarily distinct colors in $J_{n}$, each vertex of a tournament $T$ on six vertices such that $J_{n} \rightarrow T$ must have at least two distinct predecessors and at least two distinct successors. There exist fifty-six

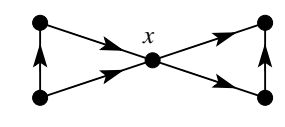

Fig. 17: The graph $H$. non-isomorphic tournaments on six vertices, but only five are such that $d^{-}(u) \geq 2$ and $d^{+}(u) \geq 2$ for each vertex $u$ : these are the ones depicted on Fig. 15 Therefore, if $\chi_{o}\left(J_{n}\right)=6$, then $J_{n}$ has necessarily a $T$-oriented-coloring for $T \in\left\{T_{6}^{1}, T_{6}^{2}, T_{6}^{3}, T_{6}^{4}, T_{6}^{5}\right\}$.

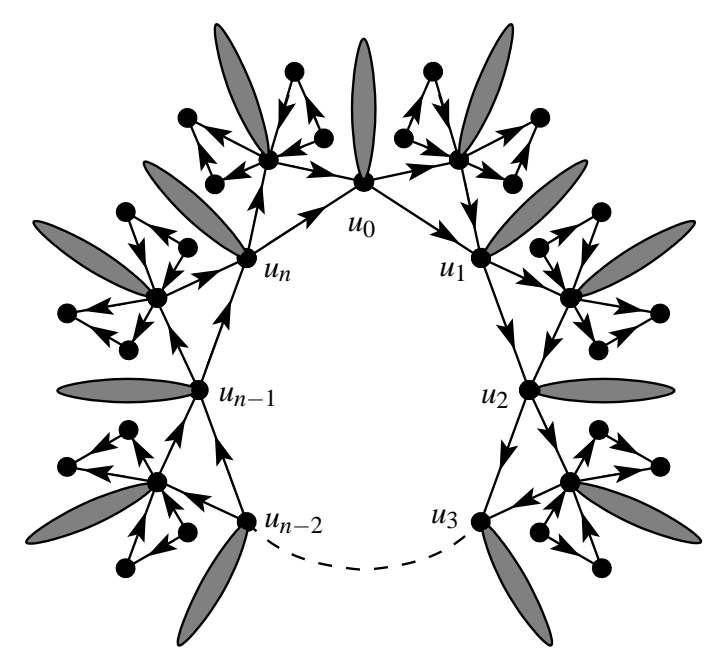

Fig. 18: An outerplanar graph $G_{n}$ without cycles of lengths 4 to $n$ such that $\chi_{o}\left(G_{n}\right)=7$ for all $n \geq 5, n$ odd, $n \not \equiv 4(\bmod 5)$. 
Let $G_{n}$ be the outerplanar graph depicted on Fig. 18 constructed as follows. We take $J_{n}, 2 n+2$ copies of $H_{n}^{\prime}$ and we identify $w_{0}$ of each copy of $H_{n}^{\prime}$ with each vertex of $W$. Since the six colors are necessarily used on the vertices of $W, G_{n} \nrightarrow T$ for all tournaments $T$ of Fig. 15 by Lemma 5.2 We thus get $\chi_{o}\left(G_{n}\right) \geq 7$. Moreover, Sopena [13] proved that every outerplanar graph has an oriented chromatic number at most 7. Therefore, $\chi_{o}\left(G_{n}\right)=7$.

\section{Proof of Theorem 1.12}

In this section, we prove that every oriented planar graph without cycles of lengths 4 or 6 has a homomorphism to the Cayley graph $Q R_{19}$.

Let us define the partial order $\preceq$. Let $n_{3}(G)$ be the number of $\geq 3$-vertices in $G$. For any two graphs $G_{1}$ and $G_{2}$, we have $G_{1} \prec G_{2}$ if and only if at least one of the following conditions hold:

- $G_{1}$ is a proper subgraph of $G_{2}$.

- $n_{3}\left(G_{1}\right)<n_{3}\left(G_{2}\right)$.

Note that this partial order is well-defined, since if $G_{1}$ is a proper subgraph of $G_{2}$, then $n_{3}\left(G_{1}\right) \leq n_{3}\left(G_{2}\right)$. So $\preceq$ is a partial linear extension of the subgraph poset.

Let $H$ be a minimal counterexample to Theorem 1.12 according to $\prec$.

As in the previous section, we proceed by reduction of configurations and discharging procedure.

\subsection{Structural properties of $H$}

Claim 6.1 The counterexample $H$ does not contain:

(C1) a 1-vertex;

(C2) a 2-vertex incident to a 3-face;

(C3) a 2-vertex adjacent to $a \leq 3$-vertex;

(C4) a 3-vertex;

(C5) a 4-vertex adjacent to two 2-vertices;

(C6) a 5-vertex adjacent to three 2-vertices;

(C7) a $k$-vertex adjacent to $(k-1) 2$-vertices with $6 \leq k \leq 9$.

Proof:

(C1) Trivial.

(C2) Consider Configuration (a) depicted in Fig.19. Any $Q R_{19}$-coloring $\gamma$ of $H \backslash\{x\}$ satisfies $\gamma\left(u_{1}\right) \neq$ $\gamma\left(u_{2}\right)$, and can be extended to $H$ by Property $P_{2,4}$. 


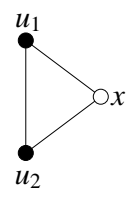

(a)

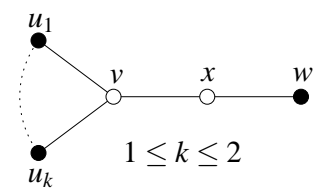

(b)

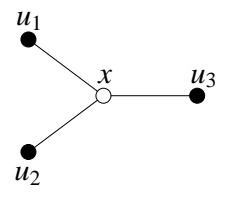

(c)

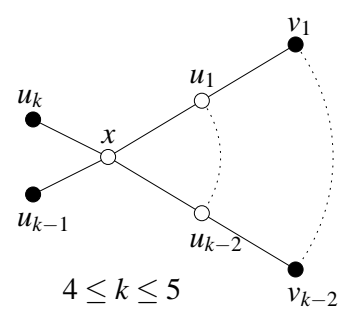

(d)

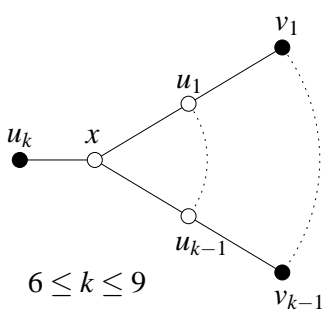

(e)

Fig. 19: Forbidden configurations of Theorem 1.12

(C3) Consider Configuration (b) $]$ depicted in Fig. 19 Any $Q R_{19}$-coloring $\gamma$ of $H \backslash\{x\}$ can be modified such that $\gamma(v) \neq \gamma(w)$ thanks to Property $P_{2,4}$. This modified coloring can be extended to $H$ by Property $P_{2,4}$.

(C4) Consider Configuration (c) depicted in Fig. 19. Notice that $u_{1}, u_{2}$, and $u_{3}$ are ${ }^{2} 3$-vertices since Configuration (b) is forbidden. If $d^{-}(x)=0$ or $d^{+}(x)=0$, we can extend any $Q R_{19}$-coloring of $H \backslash\{x\}$ to $H$ by Property $P_{3,1}$. Now, there remains two equivalent cases: (1) $d^{-}(x)=1$ and $d^{+}(x)=2$ or (2) $d^{-}(x)=2$ and $d^{+}(x)=1$. We only treat Case (1). Let us set $\Gamma^{-}(x)=\left\{u_{1}\right\}$, $\Gamma^{+}(x)=\left\{u_{2}, u_{3}\right\}$. We now consider the graph $H^{\prime}$ obtained from $H \backslash\{x\}$ by adding directed 2-paths joining respectively $u_{1}$ and $u_{2}$, and $u_{1}$ and $u_{3}$. Notice that if $H$ is a planar graph without cycles of lengths 4 or 6 , then $H^{\prime}$ is a planar graph without cycles of lengths 4 or 6 . Moreover $H^{\prime} \prec H$ since $n_{3}\left(H^{\prime}\right)=n_{3}(H)-1$. Any $Q R_{19}$-coloring $\gamma$ of $H^{\prime}$ induces a coloring of $H \backslash\{x\}$ such that $\gamma\left(u_{1}\right) \neq \gamma\left(u_{2}\right)$ and $\gamma\left(u_{1}\right) \neq \gamma\left(u_{3}\right)$, which can be extended to $H$ by Property $P_{3,1}$.

(C5)-(C6) Consider Configuration (d) depicted in Fig. 19. Any $Q R_{19}$-coloring $\gamma$ of $H \backslash\left\{u_{1}, \ldots, u_{k-2}\right\}$ can be modified such that $\gamma(x) \notin\left\{\gamma\left(v_{1}\right), \ldots, \gamma\left(v_{k-2}\right)\right\}$ thanks to Property $P_{2,4}$. This modified coloring can be extended to $H$ by Property $P_{2,4}$.

(C7) Consider Configuration (e) depicted in Fig. 19. Any $Q R_{19}$-coloring $\gamma$ of $H \backslash\left\{u_{1}, \ldots, u_{k-1}\right\}$ can be modified such that $\gamma(x) \notin\left\{\gamma\left(v_{1}\right), \ldots, \gamma\left(v_{k-1}\right)\right\}$ thanks to Property $P_{1,9}$. This modified coloring can be extended to $H$ by Property $P_{2,4}$. 


\subsection{Discharging procedure}

Lemma 6.2 Let $H$ be a connected plane graph. Then we have the following:

$$
\sum_{v \in V(H)}(3 d(v)-10)+\sum_{f \in F(H)}(2 d(f)-10)=-20
$$

Proof: Euler's formula $|V(G)|-|A(G)|+|F(G)|=2$ can be rewritten as $(6|A(G)|-10|V(G)|)+$ $(4|A(G)|-10|F(G)|)=-20$. This identity and the relation $\sum_{v \in V} d(v)=\sum_{f \in F} d(f)=2|A(G)|$ complete the proof.

We define the weight function $\omega$ by $\omega(x)=3 \cdot d(x)-10$ if $x \in V(H)$ and $\omega(x)=2 \cdot d(x)-10$ if $x \in F(H)$. It follows from Identity $[13$ that the total sum of weights is equal to -20 . In what follows, we will define discharging rules (R1) to (R6) and redistribute weights accordingly. Once the discharging is finished, a new weight function $\omega^{*}$ is produced. However, the total sum of weights is fixed by the discharging rule. Nevertheless, we can show that $\omega^{*}(x) \geq 0$ for all $x \in V(H) \cup F(H)$. This leads to the following obvious contradiction:

$$
0 \leq \sum_{x \in V(H) \cup F(H)} \omega^{*}(x) \leq \sum_{x \in V(H) \cup F(H)} \omega(x)=-20<0
$$

That demonstrates that no such counterexample can exist.

A 4-vertex is weak if it is incident to a unique 3-face and adjacent to a unique 2-vertex. The edge incident to a weak 4-vertex which is not on the boundary of the 3-face and not incident to the 2-vertex is called special. A special edge can be incident to two weak 4-vertices. Let $m_{3}(v)$ be the number of 3-faces incident to $v$. Let $m_{7}(v)$ be the number of $\geq 7$-faces incident to $v$.

The discharging rules are defined as follows (note that the initial weight of every edge of $H$ is null):

(R1) Every $\geq 7$-face gives $\frac{4}{7}$ to each edge on its boundary.

(R2) Every $\geq 4$-vertex gives $\frac{16}{21}$ to each incident 3 -face.

(R3) Every special edge gives $\frac{2}{7}$ to each incident weak 4-vertex.

(R4) Every edge on the boundary of a 3-face $f$ gives $\frac{4}{7}$ to $f$.

(R5) Let $v$ be a 2-vertex.

(R5.1) If $m_{7}(v)=2$, then the vertices adjacent to $v$ give each $\frac{6}{7}$ to $v$.

(R5.2) If $m_{7}(v)=1$, then the vertices adjacent to $v$ give each $\frac{10}{7}$ to $v$.

(R5.3) If $m_{7}(v)=0$, then the vertices adjacent to $v$ give each 2 to $v$.

(R6) Every edge incident to a 2-vertex $v$ gives the total weight obtained by (R1) to $v$. 
Let $f$ be an $l$-face. Then $l \neq 4$ and $l \neq 6$ by hypothesis.

- If $l=3, \omega(f)=-4$. Since there are no 2-vertices incident to a 3-face by (C2) and no 3-vertices by (C3), $f$ is incident to three $\geq 4$-vertices and receives from each $\frac{16}{21}$ by (R2). Moreover, each edge of its boundary gives $\frac{4}{7}$ to it by (R1). Hence, $\omega^{*}(f)=-4+3 \cdot \frac{16}{21}+3 \cdot \frac{4}{7}=0$.

- If $l=5, \omega(f)=\omega^{*}(f)=0$.

- If $l \geq 7, \omega(f)=2 \cdot l-10$. By (R1), $f$ gives $\frac{4}{7}$ to each edge on its boundary. Hence, $\omega^{*}(f)=$ $2 \cdot l-10-\frac{4}{7} \cdot l=\frac{10}{7} \cdot l-10 \geq 0$.

Let $v$ be a $k$-vertex. Then $k \neq 1$ and $k \neq 3$ by (C1) and (C 3 ).

- If $k=2$, then $\omega(v)=-4$. Observe that there are no 1-vertices by (C11, no two adjacent 2-vertices by (C 3 , and no 3 -vertices by (C4). If $m_{7}(v)=2$, then $v$ receives $4 \cdot \frac{4}{7}$ from its incident edges by (R6) and $2 \cdot \frac{6}{7}$ from its adjacent vertices by (R5.1). Hence, $\omega^{*}(v)=-4+4 \cdot \frac{4}{7}+2 \cdot \frac{6}{7}=0$. If $m_{7}(v)=1$, then $v$ receives $2 \cdot \frac{4}{7}$ from its incident edges by (R6) and $2 \cdot \frac{10}{7}$ from its adjacent vertices by (R5.2). Hence, $\omega^{*}(v)=-4+2 \cdot \frac{4}{7}+2 \cdot \frac{10}{7}=0$. Finally, if $m_{7}(v)=0$, then $v$ receives nothing from its incident edges and $2 \cdot 2$ from its adjacent vertices by (R5.3). Hence, $\omega^{*}(v)=-4+2 \cdot 2=0$.

- If $k=4$, then $\omega(v)=2$. By (C5), $v$ is adjacent to at most one 2-vertex. First, suppose that $v$ is not weak. If $m_{3}(v)=2$, then $v$ gives $2 \cdot \frac{16}{21}$ by (R2) and $\omega^{*}(v)>0$. If $v$ is adjacent to a 2-vertex, it gives at most 2 by (R5). Finally, suppose that $v$ is weak. Observe that since a 3 -face is adjacent to $\geq 7$-faces, then the 2 -vertex adjacent to $v$ is incident to at least one $\geq 7$-face. So, $v$ gives at most $\frac{10}{7}$ to the adjacent 2 -vertex by (R5) and $\frac{16}{21}$ to the 3 -face by (R2). Now, $v$ receives $\frac{2}{7}$ from its incident special edge by (R3). Finally, $\omega^{*}(v)=2-\frac{10}{7}-\frac{16}{21}+\frac{2}{7}=\frac{2}{21}>0$.

- If $k=5, \omega(v)=5$. By (C6), $v$ is adjacent to at most two 2-vertices and so $\omega^{*}(v) \geq 5-2 \cdot 2-\frac{16}{21}>0$ by (R2) and (R5).

- If $k=6, \omega(v)=8$. By (C7), $v$ is adjacent to at most four 2-vertices. If $v$ is adjacent to at most three 2-vertices, then $\omega^{*}(v) \geq 8-3 \cdot 2-\frac{16}{21}>0$ by (R2) and (R5). Now suppose that $v$ is adjacent to four 2-vertices. If $v$ is not incident to a 3-face then $\omega^{*}(v) \geq 8-4 \cdot 2=0$ by (R5). If $v$ is incident to a 3 -face, then two of the 2 -vertices are incident to at least one $\geq 7$-face. Hence, $\omega^{*}(v) \geq 8-2 \cdot 2-2 \cdot \frac{10}{7}-\frac{16}{21}>0$ by (R2) and (R5).

- If $7 \leq k \leq 9, \omega(v)=3 \cdot k-10$. By (C7), $v$ is adjacent to at most $(k-2)$ 2-vertices. Hence, $\omega^{*}(v) \geq 3 \cdot k-10-(k-2) \cdot 2-\frac{16}{21}=k-6-\frac{16}{21}>0$.

- If $k \geq 10, \omega(v)=3 \cdot k-10$. Hence, $\omega^{*}(v) \geq 3 \cdot k-10-2 \cdot k \geq 0$.

Finally, it is easy to observe that the remaining charge on each edge is non-negative.

Thus, we obtain the following contradiction which completes the proof:

$$
0 \leq \sum_{x \in V(H) \cup F(H)} \omega^{*}(x) \leq \sum_{x \in V(H) \cup F(H)} \omega(x)=-20<0
$$




\section{Concluding remarks}

Several papers dealing with the strong oriented chromatic number of planar graphs get upper bounds as corollaries of results on the maximum average degree. However, allowing triangles increases the maximum average degree as shown by Lemma 1.8 Indeed, the maximum average degree of planar graphs with given girth tends to 2 when the girth grows, whereas the maximum average degree of planar graphs without cycles of lengths 4 to $i$ tends to 3 when $i$ grows. Therefore, one might expect that the strong oriented chromatic number would increase together with the maximum average degree. Nevertheless, our results show that allowing triangles does not increase that much the strong oriented chromatic number. Consequently, the maximum average degree is not a pertinent parameter to bound the strong oriented chromatic number of planar graphs without cycles of lengths 4 to $i, i \geq 4$. Indeed, the proofs for upper bounds in this paper do not use the maximum average degree but have to use the planar structure of the graphs.

In a companion paper [9], we continue this study and prove that $\chi_{s}(G) \geq 11$ (resp. 19,43) for planar graphs without cycles of lengths 4 to 9 (resp. without cycles of lengths 4 and 5, without cycles of length 4). The proofs are assisted by computer.

Up to now, we know that for all $i \geq 4$, there exist outerplanar graphs $G$ without cycles of lengths 4 to $i$ with $\chi_{s}(G)=7$. It would be interesting to construct lower bounds for small values of $i$ to determine the relevance of our upper bounds.

\section{Acknowledgments}

We are grateful to the anonymous referees for their helpful comments which improve the presentation of the paper. We also would like to thank Éric Sopena who brought the problem to our attention. 


\section{References}

[1] O. V. Borodin and A. O. Ivanova. An oriented 7-colouring of planar graphs with girth at least 7. Sib. Electron. Math. Reports, 2:222-229, 2005.

[2] O. V. Borodin and A. O. Ivanova. An oriented colouring of planar graphs with girth at least 4. Sib. Electron. Math. Reports, 2:239-249, 2005.

[3] O. V. Borodin, A. O. Ivanova, and A. V. Kostochka. Oriented 5-coloring of sparse plane graphs. J. Applied and Industrial Mathematics, 1(1):9-17, 2007.

[4] O. V. Borodin, A. V. Kostochka, J. Nešetřil, A. Raspaud, and É. Sopena. On the maximum average degree and the oriented chromatic number of a graph. Discrete Math., 206:77-89, 1999.

[5] E. Fried. On homogeneous tournaments. In Combinatorial Theory and its Applications II (Proc. Colloq., Balatonfüred, 1969), pages 467-476. North-Holland, Amsterdam, 1970.

[6] T. R. Jensen and B. Toft. Graph Colouring Problems, chapter 4.9, pages 86-89. John Wiley \& Sons, Inc., Wiley Interscience edition, 1995.

[7] A. V. Kostochka, É. Sopena, and X. Zhu. Acyclic and oriented chromatic numbers of graphs. J. Graph Theory, 24:331-340, 1997.

[8] T. H. Marshall. Antisymmetric flows on planar graphs. J. Graph Theory, 52(3):200-210, 2006.

[9] M. Montassier, P. Ochem, and A. Pinlou. Strong oriented chromatic number of planar graphs without cycles of specifics lengths. In IV Latin-American Algorithms, Graphs and Optimization Symposium, Elect. Notes in Discrete Math., to appear, 2008.

[10] J. Nešetřil and A. Raspaud. Antisymmetric flows and strong colourings of oriented graphs. Annales de l'Institut Fourier, 49:1037-1056, 1999.

[11] P. Ochem. Oriented colorings of triangle-free planar graphs. Inform. Process. Lett., 92:71-76, 2004.

[12] R. Šámal. Antisymmetric flows and strong oriented coloring of planar graphs. Discrete Math., 273(1-3):203-209, 2003.

[13] É. Sopena. The chromatic number of oriented graphs. J. Graph Theory, 25:191-205, 1997.

[14] É. Sopena. Oriented graph coloring. Discrete Math., 229(1-3):359-369, 2001.

[15] D. R. Wood. Acyclic, star and oriented colourings of graph subdivisions. Discrete Math. Theoret. Comput. Sci., 7(1):37-50, 2005. 